\title{
Dynamic Stress And Pore Pressure Concentration For A Cavity Embedded In Saturated Soil Due To The Scattering Wave Field
}

\author{
HE Xi-ping \\ Evaluation Center of Education and Teaching, \\ Nanchang Institute of Technology, Jiangxi Nanchang \\ 330099, China \\ hxp@nit.edu.cn
}

\section{XU Man-qing}

School of Civil Engineering and Architecture, Nanchang Institute of Technology, Jiangxi Nanchang 330099, China

xmq418@163.com.cn

\author{
Huang Xiao-qiang \\ School of Civil Engineering and Architecture, \\ Nanchang Institute of Technology, Jiangxi Nanchang \\ 330099, China \\ HuangXQ@nit.edu.cn \\ Yang Zhao-ting \\ School of Civil Engineering and Architecture, \\ Nanchang Institute of Technology, Jiangxi Nanchang \\ 330099, China \\ Yangzt@nit.edu.cn
}

\begin{abstract}
According to the boundary integral equations, numerical simulation of wave scattering around a cavity with a circular cross-section embedded in saturated soil is obtained using integral transform methods. Examples dealing with the problems of wave scattering and dynamic stress concentration of $P$ harmonic plane waves by a circular cavity in a half-space poroelastic soil medium were solved, and the proposed methodology was discussed. The numerical results of this study show that the dynamic stress concentration and pore pressure concentration are influenced by the degree of fluid-solid coupling as well as the pore compressibility and water permeability of saturated soil. With increased degree of fluid-solid coupling, the dynamic stress concentration improves from 1.87 to 3.42 and the scattering becomes more significant. Under the same conditions, the dynamic stress concentration factor in the case of an impermeablepermeable boundary condition is larger than that for the permeable boundary.
\end{abstract}

Keywords- boundary integral equations; saturated soil; wave scattering; dynamic stress concentration; Green's function

\section{INTRODUCTION}

Research on the elastic wave scattering problems of underground cavities (such as tunnels and culvert structures) is important in geophysics and civil engineering both in theory and practical application. At present, studies on the elastic wave scattering problems of the holes in a half-space single-phase elastic medium is comparatively perfect. Lee and Karl [1] calculated the deformations of a hole in a half-space subjected to a steady-state $\mathrm{P}$ wave using the wave function expansion method and a curve approach instead of a linear approach. El-Akily and Datta [2] used continuous reaction and the matched asymptotic expansion method to study the dynamic responses of the cylindrical shell and pile in a half-space. A two-phase saturated soil medium is more consistent with the project practice than the single-phase medium. A solid basis for analytical study of the dynamic behavior of fluid-saturated porous elastic solids was provided by Biot $[3,4]$, who introduced his equations of motion and constitutive equations for poroelastic solids in 1956. Some scholars investigated the scattering of waves by inclusions embedded in a saturated soil medium based on Biot's porous medium dynamics theory. Deresiewicz and Rice [5] characterized wave scattering at the plane boundaries of a poroelastic region using Biot's equations. Stoll and Bryan [6,7], as well as Norris [8] characterized the wave attenuation mechanisms in a poroelastic medium and demonstrated how these effects may be incorporated into the Biot model. Hamilton [9] studied the depth dependence of wave attenuation in seafloor sediment. Mei et al. [10] introduced a simple method for a circular cavity with arbitrary radius in a poroelastic medium and for both $\mathrm{P}$ and $\mathrm{SV}$ incident waves using the boundary layer approximation. Zimmerman and Stern [11] studied the problem of wave diffraction by a spherical cavity in an infinite poroelastic soil medium using the boundary element method (BEM). Manolis and Beskos [12] presented an integral formulation in the Laplacetransformed domain to solve the problems of wave scattering by tunnels in infinite poroelastic saturated soils. Kattis et al. [13] used the BEM to solve the problem of incident harmonic $\mathrm{P}$ and SV plane waves by tunnels in poroelastic soil media with infinite extent.

In this paper, A boundary element integral equation for wave scattering by a cavity embedded in saturated 
soils was established. Examples dealing with the problems of wave scattering and dynamic stress concentration of $\mathrm{P}$ harmonic plane waves by a circular cavity in a half-space poroelastic soil medium were solved, and the proposed methodology was discussed. The numerical results are found to be in excellent agreement with the existing results.

\section{BOUNDARY INTEGRAL EQUATIONS FOR WAVE SCATTERING}

As shown in Fig .1, due to the hole embedded in saturated soils, elastic waves produce wave scattering phenomena on the cavity surface. The saturated soil total displacement $u_{i}$ can be expressed by the free wave field displacement $u_{i}^{f}$ and scattering wave field displacement $u_{i}^{s}$ caused by the discontinuous interface as follows:

$$
u_{i}=u_{i}^{f}+u_{i}^{s}
$$

The free wave field displacement $u_{i}^{f}$ is expressed as the non-existence of a half-space wave field with an irregular shape, which can be written as $u_{i}^{f}=u_{i}^{i}+u_{i}^{r}$.

The boundary integral equation for the scattering field $u_{i}^{s}$ can be obtained as follows:

$$
\begin{gathered}
c_{i j} \bar{u}_{i}^{s}(x, \omega)=\int_{s_{1}+s_{2}}\left[\bar{U}_{i j}^{G s}(x, \zeta, \omega) \bar{t}_{f}^{s}(\zeta, \omega)\right. \\
\left.-\bar{T}_{i j}^{G s}(x, \zeta, \omega) \bar{u}_{j}^{s}(\zeta, \omega)\right] d S(\zeta)
\end{gathered}
$$

where $S_{1}$ and $S_{2}$ denote half plane surface and cavity surface, respectively. As shown in Fig.1.

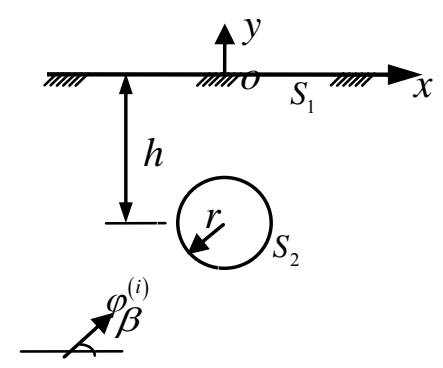

Figure 1. Model for the problem of a hole embedded in saturated soil subjected to elastic wave

For the half-plane, the boundary conditions are as follows:

$$
\begin{gathered}
\bar{t}_{j}=\bar{t}_{j}^{f}=\bar{t}_{j}^{s}=0\left(\text { on } S_{1}\right) \\
\bar{t}_{j}=\bar{t}_{j}^{f}+\bar{t}_{j}^{s}=0\left(\text { on } S_{2}\right) \\
\bar{p}_{f}=0 \text { (permeable boundary) } \\
\bar{w}_{n}=0(\text { impermeable boundary) }
\end{gathered}
$$

Considering the above boundary conditions, the boundary integral equation (2) can be written as follows:

$$
\begin{gathered}
c_{i j} \bar{u}_{i}^{s}(x, \omega)=\int_{s_{2}} \bar{U}_{i j}^{G s}(x, \zeta, \omega) \bar{t}_{j}^{f}(\zeta, \omega) d S(\zeta) \\
\quad+\int_{s_{1}+s_{2}} \bar{T}_{i j}^{G s}(x, \zeta, \omega) \bar{u}_{j}^{s}(\zeta, \omega) d S(\zeta)
\end{gathered}
$$

where $\bar{t}_{j}^{f}$ can be obtained by $\bar{u}_{i}^{f}[18]$.

\section{NUMERICAL RESULTS}

The calculation model in Fig .1 shows a circular hole with radius $r$ and buried depth $h$ embedded in half-space saturated soil. The incident wave is assumed to be a steady-state plane compression fast wave, $\varphi_{f}^{(i)}=\varphi_{f_{0}} e^{\mathrm{i} k f(x \sin \beta+y \cos \beta)} e^{-\mathrm{i} \omega t}$, in which $\beta$ is the angle of incidence and $\varphi_{f_{0}}$ is the amplitude of potential function of the wavefront by origin. In this section, the present method is used to investigate the influence of saturated soil parameters on the hole stress and pore pressure concentration coefficient with the cave border conditions of impermeablilty and permeability. The other incident wave can be discussed similarly.

The dynamic stress concentration factor is defined as the ratio of tangential effective stress to the maximum amplitude of the incident effective stress at the same point, that is, $\sigma^{*}=\sigma_{\theta} / \sigma_{0}, \quad$ where $\sigma_{0}=\operatorname{Re}\left[-(\lambda+2 \eta) k_{f}^{2} \varphi_{0}\right]$. In case of an impermeable condition, the pore pressure concentration factor is defined as the ratio of the pore pressure on the boundary of the cavity to the maximum amplitude of pore pressure at the same point, that is, $p_{f}^{*}=p_{f} / p_{0}$, where $p_{0}=\operatorname{Re}\left(-A_{f} k_{f}^{2} \varphi_{0}\right)$.

The influences of the cavity dynamic stress concentration factor and pore pressure concentration factor on the saturated soil parameters $\alpha$ and $M$, as well as the incident wave number with impermeable and permeable boundary conditions are presented in this section. The cavity is embedded on a saturated poroelastic half-space subjected to an incident wave $P$ with an incident angle $\beta=90^{\circ}$ and dimensionless wave number $\operatorname{Re}\left(k_{f} r\right) . r$ is the radius of the cavity, and the buried depth is $h=2.0 r$. The parameters of this medium are $\mu=1.0 \times 10^{7} \mathrm{~N} / \mathrm{m}^{2}$, $\rho_{s}=2.5 \times 10^{3} \mathrm{~kg} / \mathrm{m}^{3} \quad, \quad \phi=0.35$ $b_{p}=1.94 \times 10^{6} \mathrm{~kg} / \mathrm{m}^{3} \cdot \mathrm{s} \quad$, and $v=0.35$. The parameters $\alpha, M$, and $\operatorname{Re}\left(k_{f} r\right)$ assume the following values: $\quad \alpha=0.99, \operatorname{Re}\left(k_{f} r\right)=0.25$, and $M=1.0 \times 10^{8} \mathrm{~N} / \mathrm{m}^{2}$. However, when one of them changes, the others remain invariable.

Fig .2 shows the distributions of the dynamic stress concentration factors along the boundary of the cavity under permeable and impermeable conditions at the saturated soil parameters $\alpha=0.4,0.6$, and 0.99 . The saturated soil parameter $\alpha(\phi \leq \alpha \leq 1)$ is the coupling degree of a flow-solid two-phase medium, and $\alpha=\phi$ denotes completely uncoupled. Therefore, based on Fig .3(a), the dynamic stress concentration factor $\sigma^{*}$ 
increases with increased $\alpha$. The scattering effect of the cavity is enhanced under the permeable condition. Fig .2(b) shows that the dynamic stress concentration factor $\sigma^{*}$ is maximum at the hole upper apex when $\alpha$ is small; it also increases with increased $\alpha$ under the cavity boundary impermeable condition. A comparison of Figures 2(a) and 2(b) shows that the hole boundary impermeable dynamic stress concentration factor for the impermeable boundary case is larger than that for the permeable case under the same conditions.

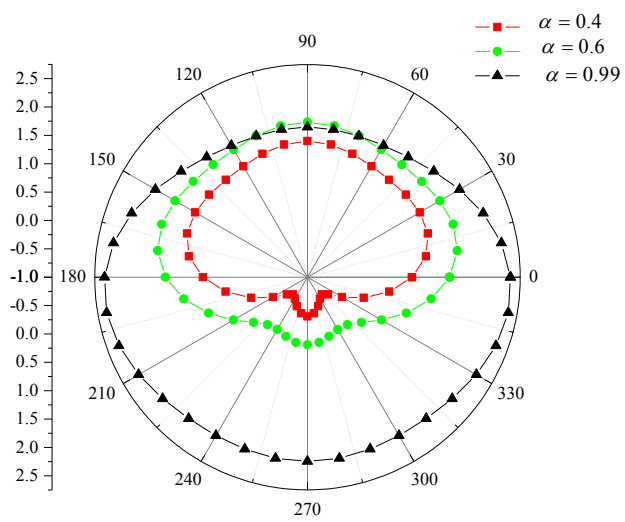

(a)

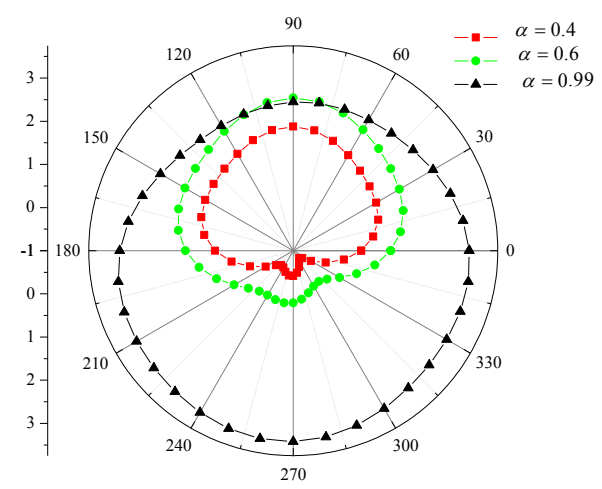

(b)

Figure 2. Influence of $\alpha$ on the dynamic stress concentration factor around a circular cavity:(a) cavity boundary permeable and (b) cavity boundary impermeable.

Fig .3 shows the influence of different incident wave numbers $\left[\operatorname{Re}\left(k_{f} r\right)=0.25,0.5,1.0,1.5\right]$ on the dynamic stress concentration factor around a cavity. It follows from Fig . 3 that the dynamic stress concentration factor decreases with increased incident wave field wave number $\operatorname{Re}\left(k_{f} r\right)$. With increased wave number, the dynamic stress concentration factors around a cavity boundary become more complex

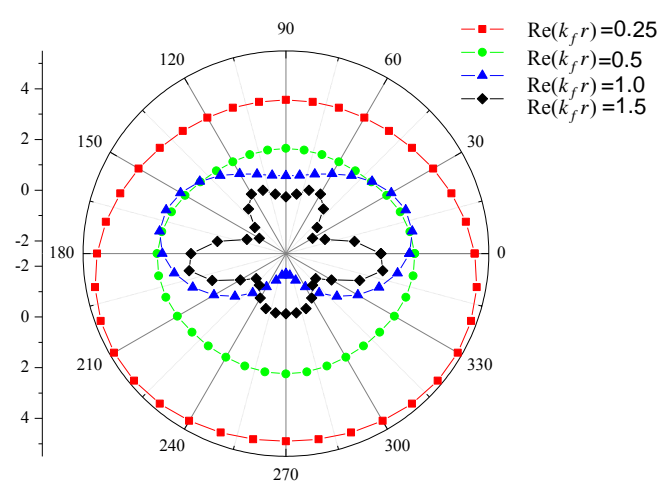

(a)

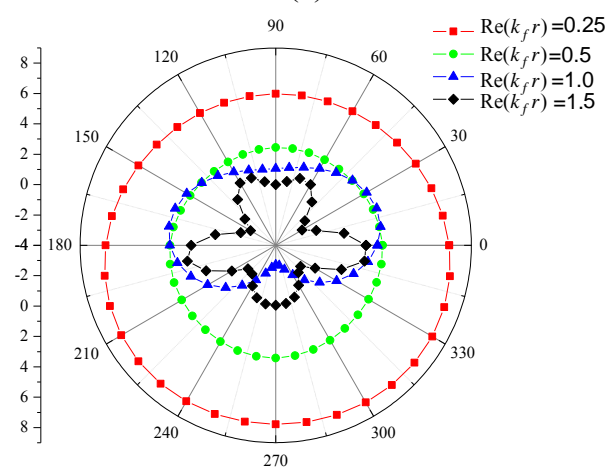

(b)

\begin{abstract}
Figure 3. Influence of $\operatorname{Re}\left(k_{f} r\right)$ on the dynamic stress concentration around a circular cavity:(a)cavity boundary permeable and (b)cavity boundary impermeable.
\end{abstract}

Fig .4 shows the variation of the dynamic stress concentration factors around a cavity versus the saturated soil parameter $M$ for three cases ( $M=$ $1.0 \times 10^{6} \mathrm{~N} / \mathrm{m}^{2}, 1.0 \times 10^{8} \mathrm{~N} / \mathrm{m}^{2}$, and $1.0 \times 10^{10} \mathrm{~N} / \mathrm{m}^{2}$ ). $M$ is the compressibility of the saturated soil pore. An increase in $M$ explains that the air in pores is filled with incompressible fluid. Fig .5(a) reveals that that dynamic stress concentration factor $\sigma^{*}$ increases with increased $M$, and the scattering effect of the cavity is enhanced under the cavity boundary permeable condition. Fig .5(b) shows that the dynamic stress concentration factor $\sigma^{*}$ is larger at the hole upper apex. It increases with increased $M$ increasing under the case of a cavity boundary impermeable condition.

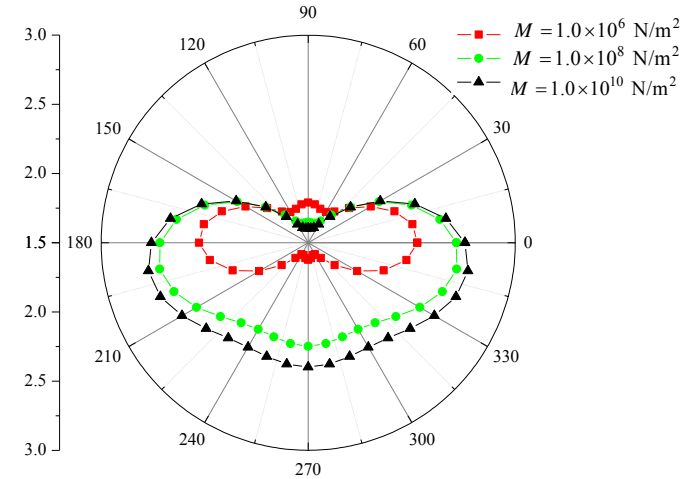

(a) 


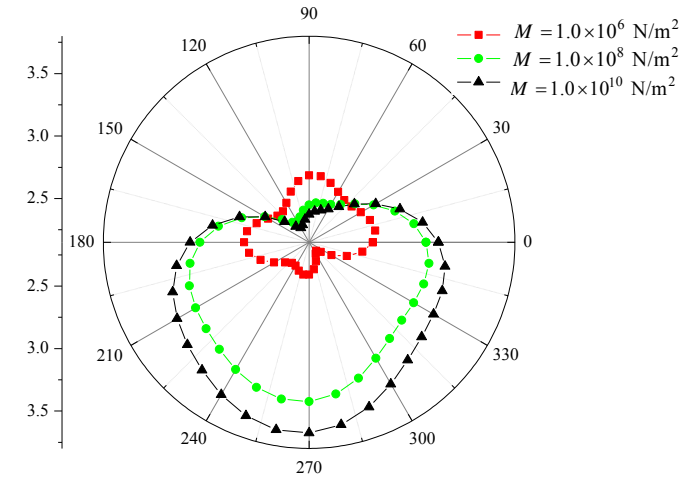

(b)

Figure 4. Influence of $M$ on the dynamic stress concentration around a circular cavity:(a) cavity boundary permeable and (b) cavity boundary impermeable.

Fig .5 shows the variation in the dynamic stress concentration factors along a cavity versus the soil parameters $\quad b_{p}=1.94 \times 10^{4} \mathrm{~kg} / \mathrm{m}^{3} \cdot \mathrm{s} \quad$, $1.94 \times 10^{6} \mathrm{~kg} / \mathrm{m}^{3} \cdot \mathrm{s}$, and $1.94 \times 10^{8} \mathrm{~kg} / \mathrm{m}^{3} \cdot \mathrm{s}$, respectively. Fig .6 shows that the dynamic stress concentration factor increases with increased $b_{p}$ for the case of the cavity boundary permeable condition.

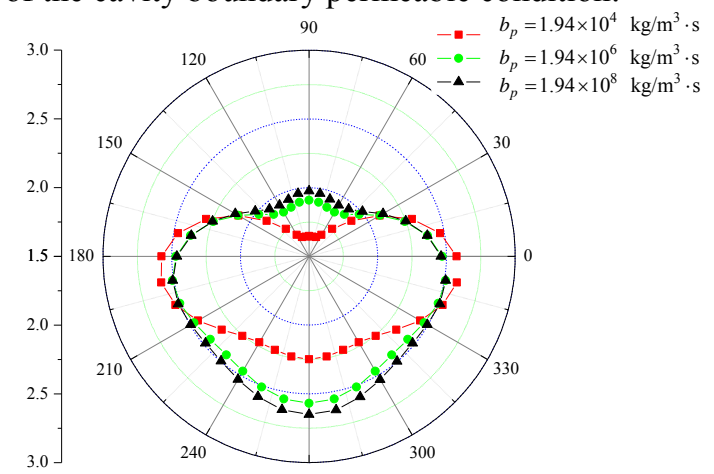

(a)

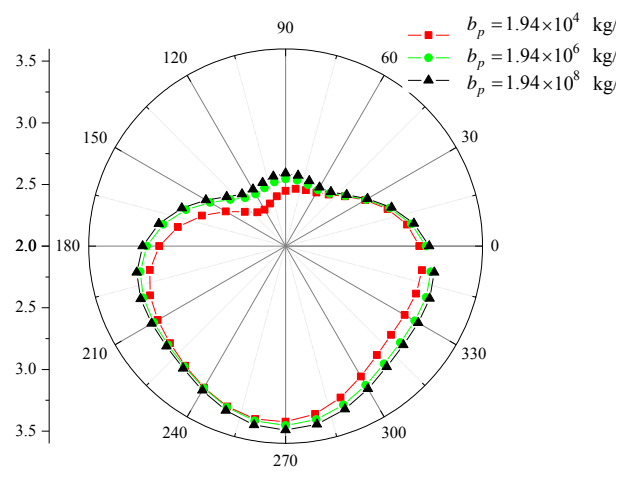

(b)

Figure 5. Influence of $b_{p}$ on the dynamic stress concentration around a circular cavity: (a) cavity boundary permeable and (b) cavity boundary impermeable.

\section{CONCLUSION}

On the basis of the preceding discussion, the following conclusions can be drawn:

(1) The numerical results of this study demonstrate that the saturated soil parameters and boundary conditions significantly influence the wave scattering along a cavity, dynamic stress concentration factor, and pore pressure concentration factor.

(2) The boundary permeability along a cavity greatly influences wave scattering. Under the same conditions, the dynamic stress concentration factor in the case of an impermeable-permeable boundary condition is larger than that for the permeable boundary.

\section{ACKNOWLEDGMENT}

The project is supported by the Jiangxi Provincial Natural Science Fund Project(NO: 20132BAB206004), Scientific Research Fund of Jiangxi Provincial Education Department(NO: GJJ14755) and the College Students' Innovation and Entrepreneurship Education Special Funds as well as the Nanchang Institute of Technology Scientific Research Training Plan for the College Students in 2013.

\section{REFERENCES}

[1] Lee V W , Karl J. Diffraction of elastic plane P waves by circular underground unlined tunnels $[\mathrm{J}]$. European Earthquake Engineering, 1993, 6(1): 29-36.

[2] El-Akily N , Datta S K. Response of a circular cylindrical shell to disturbances in a half-space[J]. Earthquake Engineering and Structural Dynamics, 1980, 8: 469-477.

[3] Biot M A. Theory of propagation of elastic waves in a fluid saturated porous solid. I. Low frequency range[J]. Journal of the Acoustical Society of America, 1956, 28(2):168-178.

[4] Biot M A. Theory of propagation of elastic waves in a fluid saturated porous solid. II. Higher frequency range[J]. Journal of the Acoustical Society of America, 1956, 28(2): 179-191.

[5] Deresiewicz H , Rice J T. The effect of boundaries on wave propagation in a liquid-filled porous solid[J]. Bulletin of the Seismological Society of America, 1962, 52(3): 595-625.

[6] Stoll R D , Bryan G M. Wave attenuation in saturated sediments[J]. Journal of the Acoustical Society of America ., 1970, 47(5), 1440-1447.

[7] Stoll R D. Theoretical aspects of sound transmission in sediments[J]. Journal of the Acoustical Society of America. 1980, 68(5), 1341-1350.

[8] Norris A N. Stoneley-wave attenuation and dispersion in permeable formations[J]. Geophysics, 1989, 54(3), 330.

[9] Hamilton E L. Sound attenuation as a function of depth in the seafloor[J]. Journal of the Acoustical Society of America . 1976 59, 528-535.

[10] Mei C C , Si B I, Cai D Y . Scattering of simple harmonic waves by a circular cavity in a fluid-filled treated poro-elastic medium[J]. Wave Motion, 1984, 6 (3), 265-278.

[11] Zimmerman C,Stern M. Scattering of plane compressional waves by spherical inclusions in a porouelastic medium $[\mathrm{J}]$. Journal of the Acoustical Society of America,1993, 94(1): 527536.

[12] Beskos D E. Boundary Element Methods in Elastodynamics[J]. Applied Mechanics Reviews (ASME) 1997,50:149-197.

Kattis S E, Beskos D E, Cheng A H D. 2D dynamic response of unlined and lined tunnels in poroelastic soil to harmonic body wave[J]. Earthquake Eng Struct Dyn., 2003,32: 97-110. 